\title{
9 Die emotionale Basis von moderner Judenfeindschaft
}

\subsection{Zur Relevanz von Emotionen bei der Analyse von Antisemitismus}

„Hatred of the Jew is not the result of a rational process. [...] Underneath [...] lies the powder keg of emotional predisposition, of a conception of the Jew which has nothing to do with facts or logic.“ (Trachtenberg 1943: 2f.)

Judenfeindschaft weist viele Dimensionen, Beweggründe und Facetten auf, hat diverse Manifestationsformen und ist in ihren konkreten Realisierungen stets eingebettet in die jeweiligen historischen Phasen zu sehen, in denen sie als religiöses, politisches, ökonomisches und/oder ideologisches Deutungsmuster in Erscheinung tritt. Bislang sind in der Antisemitismusforschung allerdings vor allem die sozial-ökonomischen und politisch-ideologischen Aspekte von Judenfeindschaft betrachtet und untersucht worden. Judenfeindschaft hat aber auch (und zwar ganz maßgeblich) eine emotionale Dimension, die wesentlich für die Erklärung judenfeindlicher Einstellungen und Aktivitäten ist. In zwei Buchtiteln des Antisemitismusforschers Robert Wistrich wird Antisemitismus treffend als „ältester Hass der Welt“ (Wistrich 1991) und „tödliche Obsession“ (Wistrich 2010) bezeichnet. Damit werden zwei wesentliche Komponenten der emotionalen Dimension von Judenfeindschaft genannt (die bei Broder 1986 mit der Phrase des „ewigen Antisemiten“ ausgedrückt werden). Diese jenseits aller Erfahrungswerte und aller Prinzipien des Verstandes zu erfassende, tief irrationale Abwehr- und Ablehnungskomponente, die im emotionalen Bewertungssystem von Menschen verankert (und als antisemitisches Ressentiment benennbar; s. hierzu Kap.4) ist, wurde bislang jedoch noch nicht ausreichend berücksichtigt bzw. detailliert analysiert (obgleich z. B. bereits Sartre 1948 in seiner phänomenologischen Abhandlung zum Antisemitismus einige wesentliche Charakteristika der emotionalen Grundlagen und der Irrationalität klar benannt ${ }^{1}$ hat; vgl. u. a. auch Trachtenberg 1943 und Adorno [1950] 1973). Es gibt natürlich psychoanalytische und sozialpsychologische Ansätze, die sich mit den Ursachen des Antisemitismus beschäftigen. Simmel ([1946] 2002b: 60) bezeichnet den Antisemitismus beispielsweise als

1 Antisemitismus beschreibt Sartre (1948: 47) als die Angst, Mensch zu sein. Er betont die mentale Konstruiertheit und Irrationalität des antisemitischen Weltbildes (Sartre 1948: 10): „Wenn es keinen Juden gäbe, der Antisemit würde ihn erfinden.“ 
„psychologisches Krankheitsbild eines Individuums“, das zur gesellschaftlichen Erklärungsvariable bzw. zum „Welterklärungsmuster“ werden kann (s. auch Berliner [1946] 2002, Hacker 1990, Staub 51995, Wahl et al. 2001, Glucksmann 2005, Hegener 2006). Ob Antisemitismus am besten als „Wahrnehmungsdefekt“, als „Paranoia“, „kollektiver Wahn“ oder „Geisteskrankheit“ einzuordnen ist (vgl. hierzu u.a. Pohl 2010), wird dabei u. a. fachspezifisch erörtert. Die kollektive emotionale Dimension von Judenfeindschaft ist aber so grundlegend und konstitutiv für dieses Phänomen, dass auch die geschichts-, sozial-, politik- und kognitionswissenschaftliche Antisemitismusforschung diese Komponente nicht ausklammern darf (s. ansatzweise hierzu auch Rensmann 2004: 129 ff., Botsch et al. 2010 und Salzborn 2010a). Dabei sollte insbesondere sehr klar gemacht werden, dass Antisemitismus keineswegs nur eine krankhafte Obsession einzelner auffälliger Extremisten und Narzissten ist, sondern sich auch bei gebildeten, feinfühligen und reflektierenden Personen findet (vgl. auch Glucksmann 2005: 85).

Emotionen wurden in der Forschung insgesamt lange als für kognitive und soziale Prozesse eher irrelevante und daher $\mathrm{zu}$ vernachlässigende Phänomene ${ }^{2}$ und die Kognition ausschließlich störende Faktoren gesehen. Die Einbeziehung emotionaler Faktoren wurde in der Kognitionswissenschaft als irrelevant oder unseriös bzw. aus methodischen Gründen auch als höchst problematisch erachtet. Allein der Versuch, Emotionen präzise zu definieren, galt lange als schwierig. Emotionen sind aber nicht etwas Diffuses, Nebulöses, wissenschaftlich nicht Erfassbares, sondern sie lassen sich in Analogie zu den Modellen der Kognition als mentale Kenntnissysteme beschreiben. Seit der emotiven Wende, die zunächst maßgeblich durch Ergebnisse der Gehirnforschung bestimmt wurde, werden Gefühle nicht länger ausgeklammert, sondern als wichtige Determinanten unseres sozialen und kognitiven (Er)Lebens berücksichtigt (s. Schwarz-Friesel 2007: 15). Die menschliche Kognition wird nicht mehr als ein völlig autonomes System verstanden, das ausschließlich von Vernunft und Verstand regiert wird (s. hierzu auch Damasio 2000 und Kahneman 2011). Geistige (und soziale) Aktivitäten werden entscheidend von emotionalen Prozessen gesteuert und begleitet ${ }^{3}$

2 Die Trennung von Kognition und Emotion, von Denken und Fühlen hat eine sehr lange kulturelle Tradition: Das gesamte neuzeitliche, abendländische Menschenbild ist durch einen tiefgreifenden Dualismus hinsichtlich Geist und Gefühl geprägt. Emotionen hatten daher in Alltags- und Wissenschaftskonzeptualisierungen lange den Status irrelevanter Begleiterscheinungen und marginaler Nebeneffekte. Zur Thematisierung der Relevanz von Emotionen in der Geschichtswissenschaft s. Jensen/Morat (2008) und Frevert (2009).

3 Beim Antisemitismus dominieren die destruktiven Emotionen, die kognitive Operationen behindern, zerstören und derangieren, die Vernunft und Verstand außer Kraft setzen können sowie den Gesetzen der Logik zuwiderlaufen (s. Kap. 9.4). Nicht notwendigerweise stehen sich Emotion und Kognition jedoch strikt konträr gegenüber. Und nicht immer sind emotionale 
(s. u.a. Salovey et al. 2004 und zu ,sozioemotionalen Orientierungen“ Wahl et al. 2001: 54). Denken und Fühlen sind nicht strikt zu trennen, vielmehr weisen Gedanken und Gefühle wesentlich mehr Gemeinsamkeiten auf als Unterschiede (s. Schwarz-Friesel 2008). Die denk- und verhaltensbeeinflussende Wirkung von Emotionen erklärt sich aus ihrer Bestimmung und Definition als Kenntnis- und Bewertungssystemen. Emotionen sind mehrdimensionale, intern repräsentierte und subjektiv erfahrbare Syndromkategorien, die sich vom Individuum introspektiv-geistig sowie körperlich registrieren lassen. Ihre wichtigste Funktion besteht darin, (positive oder negative) Bewertungen $\mathrm{zu}$ vermitteln. Diese Werturteile können für andere in wahrnehmbaren Ausdrucksvarianten realisiert werden. Die Prozesse der Bewertung betreffen Einschätzungen, mit denen ein Individuum entweder sich selbst, seine Körperempfindungen, seine Handlungsimpulse, seine kognitiven Denkinhalte oder andere Menschen, ihre Verhaltensweisen und allgemeine Umweltsituationen (im weitesten Sinne) beurteilt. ${ }^{4}$ Subjektiv erfahrbar werden Emotionen als Gefühle wahrgenommen und ausgedrückt (s. Schwarz-Friesel 2007: 77 ff.).

Emotionen sind Bewertungen, die Einstellungen in Bezug auf die Welt bestimmen. Andererseits determinieren die kognitiven Einstellungen wiederum unsere Gefühle. Permanent verankerte Einstellungen sind Teil des Kenntnissystems, das im Langzeitgedächtnis (LZG) langfristig gespeichert ist. Sie sind Bestandteil unseres allgemeinen Bewertungssystems, mit dem wir die Welt, unsere Mitmenschen, ihre Handlungen etc. beurteilen. Das emotionale System speichert auch sozial vermittelte Gefühlswerte, die kollektives Wissen repräsentieren. Diese können die individuellen Einstellungen maßgeblich prägen. Das Phänomen der emotionalen Einstellungen zeigt auch die untrennbare Interaktion von emotionalem und kognitivem Kenntnissystem. Emotionale Einstellungen basieren auf kognitiven Repräsentationen und aufgrund dieser gebildeten Urteilen. Ein Vorurteil

\footnotetext{
Bewertungen irrational oder gar destruktiv. Es gibt auch positive, produktive und mit kognitiven Prozessen der Vernunft korrelierende Emotionen. Emotionale Zustände können kognitive Prozesse wie Verarbeiten, Lernen, Behalten, Erinnern optimieren und beschleunigen (s. Schwarz-Friesel 2007: $115 \mathrm{f}$.).

4 Dieser kognitivistische Emotionsansatz sieht die Bewertung als das konstitutive und zentrale Element von Emotionen an. Vgl. Scherer (2004) und Schwarz-Friesel (2007: 72 ff.). S. auch Solomon (32001: 172 f.): „Was sind Gefühle? Als fundamentale Urteile oder Urteilskomplexe bilden sie existentielle Initiativen, die [...] uns und unsere Stellung in der Welt bestimmen, Werte, Ideale, Strukturen und Mythologien entwerfen, nach denen wir uns richten und in deren Rahmen wir leben. Insofern hängen Gefühle stark von Meinungen und Überzeugungen ab. [...] Ärgern können wir uns nur in der Annahme, daß jemand uns Unrecht getan oder geschadet hat. [...] Gefühle sind wertende (oder 'normative') Urteile: über meine Lage sowie über mich und/ oder alle anderen.“
} 
gegenüber einer bestimmten Menschengruppe ist somit eine im LZG gespeicherte komplexe Stereotyprepräsentation, die an eine emotionale Negativeinstellung gekoppelt ist. Zeichnet sich eine (z. B. ideologisch determinierte) Stereotyprepräsentation durch extrem negative Merkmale aus, ist in striktem Kontrast zum eigenen Selbstkonzept ausgerichtet und stellt eine Feindbildkonstruktion dar, kann diese kognitive Repräsentation dazu führen, dass grundlegende Gefühle wie Mitleid und Mitgefühl sowie moralische Empfindungen komplett ausgeblendet werden. So kann man erklären, wie Menschen anderen Menschen ohne Skrupel Gewalt zufügen und keinerlei Empathie für die Nöte anderer haben (s. hierzu auch Welzer 2005 und Haubl/Caysa 2007).

Da Einstellungen und emotionale Bewertungssysteme nicht beobachtbare innere Zustände sind, die sich aber in den Kodifizierungsformen sprachlicher Äußerungen widerspiegeln, bietet die Sprachanalyse methodisch die Möglichkeit, über die Verbalmanifestationen Einblick in die zugrunde liegenden Bewertungsmuster zu erhalten. Wir werden uns daher im Folgenden auf die empirisch nachweisbaren Manifestationen von emotionalen Zuständen und Prozessen konzentrieren, die sich über sprachliche Ausdrücke und textuelle Argumentation rekonstruieren lassen.

Zum einen geht es also um das Emotionspotenzial ${ }^{5}$ antisemitischer Texte, das sich in zahlreichen Verbalformen zur emotionalen Befindlichkeit der Sprachproduzenten zeigt. Gefühle wie Hass, Wut, Angst, Unbehagen, Abneigung usw. werden explizit benannt und ausgedrückt. Zum anderen zeigt die Textanalyse aber auch den Einfluss von Emotionen (als irrationale Störfaktoren, die Vernunft und Logik zuwiderlaufen) auf argumentative Strukturen sowie semantisch-konzeptuelle Kohärenz: Verbal-antisemitische Texte zeichnen sich häufig durch eklatante Widersprüche, Fehlannahmen und Trugschlüsse aus.

\subsection{Das Emotionspotenzial antisemitischer Texte: Emotionsausdruck und Gefühlsbezeichnungen}

Das Emotionspotenzial der Schreiben, ${ }^{6}$ die judenfeindliche Inhalte kommunizieren, ist sehr hoch. Die Texte enthalten überdurchschnittlich viele emotionsbe-

\footnotetext{
5 Das Emotionspotenzial einer sprachlichen Äußerung ergibt sich als textinterne Eigenschaft aus allen sprachlichen Formen der Emotionsbezeichnungen und des (expliziten wie impliziten) Emotionsausdrucks (s. Schwarz-Friesel 2007: 210 f.). So lässt sich das Emotionspotenzial eines Textes als hoch oder niedrig bestimmen.

6 Die quantitative Analyse eines Subkorpus von insgesamt 4.695 Schreiben aus den Jahren 2002 bis 2006 ergab, dass in den E-Mails an die IBD knapp 70 Prozent (68,9 Prozent)
} 
zeichnende und emotionsausdrückende Lexeme. Mittels zahlreicher Verbalformen drücken die Verfasser ihre „ohnmächtige Wut und Empörung“, ihren „großen Ärger“ und „unmäßigen Zorn“ aus, wenn es um die „Verbrechen der Israelis“ oder die „freche Komplizenschaft des Zentralrats“ geht. ${ }^{7}$ Häufig thematisieren die Verfasser ein diffuses Gefühl der Aufregung und des Aufgebrachtseins und betonen ihre innere Aufgewühltheit beim Schreiben:

„Ein total unstrukuriertes Mail ich weiss aber ich bin einfach zu aufgeregt.“[IBD_12.07.2006_Fra_001]

Viele der Schreiber geben explizite Verweise wie in (2) und (3), dass ihre emotionale Situation sie nicht anders handeln lässt und dass das Bedürfnis, Juden scharf zu kritisieren und alles zu verbalisieren, was sie an Hass, Wut und Ekel empfinden, so groß ist, dass keine rationale Überlegung sie davon abhalten kann (s. hierzu auch weiter unten Bsp. (23)):

(2) „Ich habe hiermit geschrieben, was zu schreiben war. Dazu drängte es mich innerlich.“[ZJD_Gaza2009_169/816_Len_001]

(3) „in meiner jetzigen Rage scheint es mir sogar sinnvoll zu sein, dies so zu schreiben, denn ich wünsche mir, daß Sie nicht so verstockt und verbohrt sind und das mein Apell Sie so erreicht!“ [ZJD_27.07.2006_Stu_001]

Emotionen werden generell über drei grundlegende Parameter bestimmt: Wertigkeit (Positiv- oder Negativ-Emotion), Dauer (zeitliche Erstreckung des emotionalen Zustandes) und Intensität (vgl. Schwarz-Friesel 2007: 69 ff.). Bei verbalantisemitischen Texten weisen diese Parameter extreme Werte auf.

Es gibt viele verbale Indikatoren wie emphatische Phrasen in (4) und (5), dass der Parameter der Intensität bei den emotionalen Prozessen der Verfasser sehr hoch ist: „Ich bin fassungslos und tief erschüttert!!!!“[ZJD_23.11.2007_Wer_001]

emotionale Textteile, also emotionsbezeichnende oder -ausdrückende Lexeme enthalten sind. Bei den Zuschriften an den ZJD sind es 60,1 Prozent. Ab 2007 sind in über 70 Prozent der Texte an den ZJD emotionale Verbalformen enthalten.

7 Mittels emotionsbezeichnender Lexeme wie Angst, Hass, wütend referiert man explizit auf bestimmte Gefühle; mit emotionsausdrückenden Lexemen vermittelt man emotionale Zustände und Prozesse über die (wertende) Semantik der Ausdrücke (z. B. mittels affektiver Adjektive wie widerwärtig oder Modalpartikeln wie leider; als emotionsausdrückend fungieren auch Interjektionen wie $I h$ ). 
„es erschüttert mich bis ins Mark so etwas lesen zu müssen.“ [ZJD_26.07.2006_ano_011]

Der Parameter der Intensität wird zudem durch graphemische Emphasesignale (vor allem zum Teil exzessiv aneinandergereihte Ausrufezeichen), Schriftgröße und -farbe (z. B. Fettdruck) kodiert. Interjektionen, mit denen die Schreiber Missfallen, Abscheu, Ekel o. Ä. ausdrücken, werden zu Beginn oder am Ende von evaluativen Aussagen eingefügt:

$$
\text { „Pfui Israel, pfui!““ [IBD_18.05.2006_Oss_001] }
$$

Häufig findet sich in diesem Zusammenhang die Thematisierung einer Fassungsund Sprachlosigkeit angesichts des von den Verfassern als „,bedrohlich“, „entsetzlich“, „unfassbar" und „fürchterlich“ bezeichneten Geschehens im Nahen Osten (das auch den Juden in Deutschland angelastet wird wie in (8)):

„mit Fassungslosigkeit habe ich in den Nachrichten ihre vollkommen überzogene Reaktion auf die entführung ihrer Soldaten verfolgt. Ich bin über ihre Gewalt entsetzt ,mit welchem Recht zerstören Sie Brücken und Straßen und Töten Unschuldige?“[IBD_12.07.2006_Fle_001]

(8) „mir fehlen die worte,schämen sie sich für ihr land ! meine familie und ich sind entsetzt.widerlich.ekelhaft.“[ZJD_29.07.2006_Gol_001]

Solche intensiven Gefühlswerte sind normalerweise nur bei persönlicher Betroffenheit $\mathrm{zu}$ konstatieren, d. h. wenn es sich um eigene existenzielle und familiäre Angelegenheiten handelt. Die Verfasser zeigen also eine emotionale Involviertheit, die unverhältnismäßig für die Diskursform „Stellungnahme zu politischen Situationen“ ist und in keiner Relation zum Anlass und der persönlichen Situation der Schreiber steht. Diese produzieren Texte mit einem so hohen Emotionspotenzial, als ob ihnen selbst unermessliches Leid zugefügt worden sei oder als ob sie einen nahen Verwandten verloren hätten, als ob sie sich selbst in großer Not und Gefahr befänden:

(9) „Diese Worte kommen aus meiner Seele, die zerrissen ist. [...] Auch jetzt, wenn die Waffen schweigen, geht es mir noch nicht viel besser.“ [IBD_19.01.2009_Man_001]

Gerade die Korrelation von innerer Erregung bzw. Leid und Sprachlosigkeit bzw. Verbalisierungsproblemen zeigt sich für Individuen in Extremsituationen (s. 
Schwarz-Friesel 2007: 234 f., 311 ff. und 2011a), für den politischen Diskurs ist sie nicht charakteristisch. Hier sind kritische Reflexion und rationale Argumentation (trotz eventuell großer Empörung) dominant. Die große affektive Involviertheit ist ein Indikator dafür, dass keine kritische Stellungnahme, sondern eine als bedrohlich, subjektiv höchst emotional empfundene Situation für die Schreiber vorliegt, die in Kontrast zur objektiven Sachlage steht.

Das Lexem Hass ist neben Wut das in den Schreiben am häufigsten benutzte. In über 49 Prozent der Texte finden sich Varianten wie Hass, hasserfüllt, hassend etc. „Hass“ dient als Sammelbegriff für besonders aggressive Abwehr- und Feindseligkeitsgefühle. Hass ist eine heftige Abneigung gegenüber einer Person oder Gruppe und geht oft einher mit Verbitterung, Wut und als Ohnmacht empfundener Angst. Hass ist obsessiv (basiert auf einer starren, konzeptuell geschlossenen Einstellung, die alles am Gehassten zwanghaft ins Negative umdeutet und gefühlsmäßig keine Empathie zulässt). Hass manifestiert sich sprachlich keineswegs nur eruptiv und kognitiv arm an Inhalt, sondern kann (wie jede Emotion) bewusst und strategisch als Gefühl ${ }^{8}$ artikuliert werden. Man kann also in den Äußerungen zwischen einem affektiven Hass und einem rationalen Hass unterscheiden ( $\mathrm{zu}$ affektivem und rationalem Hass in antisemitischen Hass-Mails s. ausführlicher Schwarz-Friesel 2012b).

Während rechtsextremistische Schreiber ihr Gefühl direkt und ich-bezogen artikulieren wie in (10) und (11) und dies mit Beschimpfungen und Verwünschungen verknüpfen (s. hierzu Kap. 10):

$$
\begin{aligned}
& \text { „Möget Ihr leiden - Ich hasse Euch alle - !!!!!!!!!!!!!!!"“ } \\
& \text { [ZJD_Gaza2009_754/816_ano_001] }
\end{aligned}
$$

$$
\text { „Wir hassen Euch Judenschweine!“ [IBD_09.04.2006_ano_026] }
$$

„ich kann nicht beschreiben was ich für einen hass für euer land empfinde.“[IBD_13.07.2006_ano_003]

\footnotetext{
8 Gefühle sind erlebte Emotionen, d. h. subjektiv erfahrbare Bewertungen emotionaler Zustände. Die bewusste Repräsentation der Emotion setzt ihre Konzeptualisierung voraus. Damit sind Gefühle kognitiv beeinflusste Zustände, s. Scherer (2004) und Schwarz-Friesel (2007: 77 ff.). In der Emotionspsychologie unterscheidet man zwischen „hot emotion“ und „cold emotion“, d. h. verschiedenen Gefühlszuständen bzw. -prozessen einer Emotion (s. u. a. Teasdale 1999). Vgl. auch Haubl/Caysa (2007: 82): „Echter Hass muss also weder primitiv noch vorzivilisatorisch, noch unbeherrscht und irrational sein, sondern er kann vielmehr sublim, beherrscht, edel, zivilisiert, berechnend, klug und vernünftig auftreten.“
} 
deklarieren Verfasser aus der (gebildeten) Mitte ihre Aggressivität als Sorge, Trauer, Enttäuschung und Entrüstung, obgleich in den Texten deutlich erkennbar ist, dass es sich um judeophobe Abneigung handelt (s. hierzu auch Kap. 11.2).

„,ich schreibe ihnen aus Sorge um die Zivilisten, besonders um die Kinder, im Süden des Libanon.“ [IBD_26.09.2006_Fra_001]

„Ich bin sehr enttäuscht und empört [...]“ [ZJD_Gaza09_63/816_Wil_001]

Bei gebildeten Verfassern aus der Mitte ist der Hass nicht vulgär-affektiv, sondern artikuliert sich scheinbar rational. Er zeigt sich oft als kalte Wut und Verachtung in der Form eines paternalistischen Rechthabenwollens und Belehrens (s. auch die Beispiele in Kap. 10.2).

(15) „Betrachten Sie mein Schreiben bitte nicht als Angriff, sondern vielmehr als freundschaftlichen Rat.“[ZJD_07.08.2006_Hön_001]

Vielfach erkennt man eine mühsam unterdrückte Aggressivität, die nur durch Sozialformen zurückgehalten wird (s. auch Bsp. [ZJD_27.07.2006_Rau_001] im Anhang).

(16) „Als deutsche Juden sollten Sie ihren Glaubensbrüdern in Israel einen Ratschlag geben.“[ZJD_Gaza2009_98/816_Pet_001]

(17) „Wo ist Ihr Gerechtigkeitssinn wenn es um Israel und Palästina geht??? Wundern Sie sich daher nicht, wenn das jüdische Volk auch heute noch Feinde hat oder keine Freunde bekommt!“

[ZJD_Gaza2009_46/816_Bau_001]

Häufig werden emotionsbezeichnende Ausdrücke am Ende der E-Mails durch Grußformen wie „mit besorgten Grüßen“ oder „ein enttäuschter Israelfreund“, „ohne solidarische Grüße“ usw. kommuniziert. Die Schreiber aus der Mitte vermeiden Ich-Botschaften der Art Ich/wir hasse(n). Vielmehr kommunizieren sie Hassgefühle bevorzugt als generische, unpersönliche Aussagen wie „Die Welt hasst Israel!“ [IBD_04.12.2007_Dro_001] oder „So sät man Hass.“ [ZJD_Gaza09_ Dös_001]. Oft werden Emotionen auch transferiert auf Dritte; die Verfasser schreiben also (scheinbar) nicht über sich selbst, sondern über die Emotionen Dritter („Meine Freunde bekommen richtige Hassgefühle.“) und vermeiden es so, sich als hass- oder wuterfüllte Menschen zu identifizieren. Oft werden die extremen Gefühle auch mit Verweis auf mediale Berichterstattungen oder Verlautba- 
rungen des Zentralrates als kausal begründete Reaktionen der „legitimen Empörung oder Verachtung“ ausgegeben:

„Bei mir als Sozialwissenschaftler lösen Ihre Kommentare immer mehr negative Gefühle über Ihren Verein aus. Bedenken Sie, welche Folgen Ihre besserwisserischen Ausführungen auf Dauer erzeugen!“ [ZJD_16.04.2007_Sch_004]

(19) „Was man jeden Tag im Radio hört, im TV sieht und in den Zeitungen lesen muß, verursacht echte Hassgefühle. Heute morgen hörte man einen Kommentarim Radio, wo über die Zustände im besetzten Westjordanland berichtet wurde...“ [IBD_21.09.2011_Kub_001]

\section{Inszenierte Emotionalität?}

Inwieweit die im Text genannten Emotionen immer tatsächlich auch authentisch den gefühlten Emotionen entsprechen oder ob es sich um Emotionsinszenierungen handelt, die persuasiv eingesetzt werden, um z. B. das positive Selbstbild der Schreiber aufrechtzuerhalten oder den Äußerungen mehr Nachdruck zu verleihen, kann nicht eindeutig festgestellt werden. Wir stoßen hier teilweise auf das Problem der Differenzierung von tatsächlicher Emotionalität und vorgetäuschten Gefühlen. Bei der Mehrzahl der E-Mails und Briefe aber kann man davon ausgehen, dass genuine Gefühle verbalisiert sind, da die Schreiber sich unaufgefordert und ohne Öffentlichkeit an den ZJD und die IBD wenden.

Signifikant ist, dass die Verfasser aus der Mitte in ihren feindseligen Schreiben Strategien benutzen, die der Legitimierung des Hasses (der stets re-kodiert wird als „Sorge um den Weltfrieden“, „Furcht vor globalem Krieg“ oder „humanistisch geprägtes Verantwortungsgefühl“) dienen. $\mathrm{Zu}$ diesen Strategien gehört das Leugnen des eigenen Hasses (selbst dann, wenn dieser zuvor durch intensive emotions- und stereotypausdrückende Lexeme artikuliert wird), indem dies meta-kommunikativ thematisiert wird:

„Ihr seid unverbesserliche fiese eigennützige Kreaturen! Dies ist kein Hassbrief - sondern meine eigene Meinung über Euch!“

[ZJD_Gaza2009_20/816_ano_001]

Oft antizipieren die Schreiber nach ihren wütenden und hasserfüllten Verbalattacken deren Wirkungspotenzial, ohne dieses jedoch akzeptieren zu können: 
„Ach ja, ich weiß schon was jetzt kommt: Die unvermeidliche Antsemitismuskeule. Geschenkt.“[ZJD_24.07.2006_Sch_001]

Obgleich einige Verfasser die spezifische Kommunikationssituation thematisieren, sind sie nicht in der Lage, sich aus ihrem konzeptuell geschlossenen Weltbild zu lösen und kritisch zu reflektieren, was sie schreiben. Zu groß ist das Bedürfnis, die intensiven Gefühle artikulieren zu wollen:

$$
\begin{aligned}
& \text { „Diese, meine Meinung mußte ich endlich mal loswerden!!“ } \\
& \text { [ZJD_24.06.2002_Mau_001] }
\end{aligned}
$$

Ein promovierter Dauerschreiber aus Berlin thematisiert den Konflikt zwischen gesellschaftlicher Erwartungshaltung und subjektiver Gefühlslage:

$$
\begin{aligned}
& \text { „Was da speziell in den letzten Tagen des Konfliktes zwischen Libanon } \\
& \text { und Israel passiert ist, hat mir die Sprache verschlagen und mich rich- } \\
& \text { tiggehend wütend auf Juden gemacht. Ich weiß, daß man solche Wut- } \\
& \text { und Haßgefühle gegenüber Juden in diesem Land nicht äußern darf, } \\
& \text { und es mußte schon viel geschehen, bis mir der Kragen geplatzt ist.“ } \\
& \text { [ZJD_30.11.2006_Gel_001] }
\end{aligned}
$$

Die Feindseligkeit wird pseudo-objektiv begründet und argumentativ elaboriert vorgetragen. Das Hassobjekt wird zunächst transferiert: Nicht die Juden, sondern stellvertretend für diese wird der Staat Israel als „kollektiver Jude“ stigmatisiert (s. Kap. 7). Dann aber erfolgt doch die Generalisierung und generische Referenz auf alle Juden. Dies zeigt zum einen, wie das moderne Selbstbild dieser Schreiber mit der Vorstellung eines von Vorurteilen determinierten Menschen kollidiert, und zum anderen, wie tief emotional verankert und der Reflexion entzogen das anti-jüdische Ressentiment bei diesen ist (s. hierzu auch Kap. 9.4.2).

Bei der Bewertung israelischer Militäraktionen dominiert die Gefühlsartikulation von Entrüstung:

(24) „Sehr geehrte Damen und Herren, hiermit möchte ich meine Empörung und meine Abscheu über die menschenverachtende und verbrecherische Politik der israelischen Regierung zum Ausdruck bringen.“ [ZJD_31.07.2006_Sch_003]

Wut kommt ebenfalls fast immer in Hinblick auf angebliche israelische Verbrechen (und auf die unterstellte Holocaustnutznießung deutscher Juden) zum Aus- 
druck, meist in der Verbalkonstruktion macht mich/uns wütend/hat mich wütend gemacht:
„hiermit möchte ich meine Verwunderung und meine Wut über den aktuellen Krieg der Juden gegen die Moslems zum Ausdruck!“ [ZJD_28.07.2006_Ren_001]

Das Gefühl der Enttäuschung wird ebenfalls viel kommuniziert. Es impliziert immer das Element der Schuldzuweisung an den Adressaten. Zum einen wird die Dimension der Reaktivität fokussiert ('Israelis/Juden verhalten sich so böse, dass man zwangsläufig geschockt und enttäuscht ist') und zum anderen wird die eigene Emotionalität so rationalisiert ('es gibt eine Ursache bzw. einen Auslöser für die eigenen heftigen Gefühle') und legitimiert ('man kann gar nicht anders'). Die Emotionsausdrücke zu Enttäuschung, Empörung und Wut werden daher auch sehr häufig mit der expliziten oder impliziten Stereotypkodierung JUDEN SIND SELBST SCHULD, WENN MAN SIE NICHT MAG vorgebracht.

$$
\begin{aligned}
& \text { „Dabei verkennen Sie jedoch, daß gerade diese provozierende Haltung } \\
& \text { Ihrerseits immer mehr Menschen wütend macht - was bei mir nur Enttäu- } \\
& \text { schung und tiefe Sorge auslöst.“ [ZJD_27.07.2006_Stu_001] }
\end{aligned}
$$

Von den zahlreichen Adjektiven, die auf emotionale Zustände referieren, werden furchtbar und schrecklich besonders häufig benutzt, wenn die israelische Politik bewertet werden soll:

$$
\begin{aligned}
& \text { „Ich, für meine Person, bin enttäuscht, traurig und beschämt, dass } \\
& \text { gerade die Israelis, die doch eigentlich am Besten wissen sollten, wie } \\
& \text { schrecklich Rechtlosigkeit, Tyrannei und Mord sind, sich zu solch einem } \\
& \text { barbarischen Treiben hinreissen lassen konnten.“ }
\end{aligned}
$$

[ZJD_26.07.2006_Kra_001]

Die Referenzialisierungen zu Israel zeichnen sich in über 90 Prozent der E-Mails durch brachiale Verbalformen der Be- und Entwertung aus: Wörter wie Massaker, unvorstellbare Grausamkeit, willkürliches Morden, Massenmord, unverhältnismäßige Gewalt, mit denen Militäraktionen der Israelis bezeichnet werden, vermitteln über ihre Semantik drastische Perspektivierungen und Evaluationen. Sie implizieren zudem alle internale Attributionen ${ }^{9}$ in Bezug auf die Verhaltensweisen von Juden und/oder Israelis.

9 Bei der Beurteilung von menschlichem Verhalten findet immer eine kausale Attribution statt 
„Mittlerweile verstehe ich die Judenhasser. Was die Juden im Gazastreifen machen ist Massenmord.“[ZJD_Gaza2009_211/816_Eku_001]

Der Hass kommt diesen Darstellungen zufolge nicht aus dem mentalen Innenleben und der Einstellungsstruktur der Sprachproduzenten, sondern wird stets extern hervorgerufen durch das Verhalten von Juden und/oder Israelis. Somit werden diese kausal nicht nur als Verursacher von Leid und Gewalt in der Welt, sondern auch für die persönliche „Gefühlsmisere“ der Schreiber verantwortlich ${ }^{10}$ gemacht:

„Ihr allein seid an der ganzen Misere schuld. Man raubt anderen nicht den Lebensraum. Ich hasse Euch.“ [IBD_02.12.2006_ano_022]

(30) „So wie dieser Unmensch sich aufführt, kann einem nur Wut und Haß gegen Israel packen. Und da finde ich es nur gerechtfertigt, daß sich die Palästinenser mit Selbstmordattentaten wehren, denn Waffen, wie die frechen Israelis haben sie ja nicht.“[ZJD_24.06.2002_Mau_001]

Solche Emotionskodierungen stellen eine besondere Variante der Selbststilisierung der Deutschen als Opfer dar: 'die an den Verbrechen der Israelis und des unverantwortlichen Versagens des jüdischen Zentralrats leidenden Deutschen, die keine Ruhe finden', die zu Ekel, Hass und Wut „gezwungen werden“.

Auffällig oft wird bei diesen Gefühlsbekundungen (zumeist pseudo-religiös) eine metaphysische Macht angesprochen:

„Wie konnte Gott es zulassen dass wir euch ertragen muessen.“ [ZJD_23.10.2007_ano_001]

$$
\begin{aligned}
& \text { „Oh bitte Gott, richte auch über den Mörder Staat ISRAEL“ } \\
& \text { [IBD_12.07.2006_ano_002] }
\end{aligned}
$$

(s. u.a. Weiner 1986). Diese spiegelt Überzeugungen über die Ursachen von Ereignissen und Sachverhalten wider. Externale Attributionen sehen die Gründe in äußeren Faktoren (Umwelt, Notwehrsituation, Gefahr etc.), internale Attributionen dagegen ordnen Verhaltensweisen dem Charakter von Menschen zu (s. hierzu Kap. 7).

10 In diesem Sinne ist auch auf der Gefühlsebene eine Täter-Opfer-Struktur zu erkennen: Die hassenden Antisemiten werden durch die „Untaten der Juden“ gezwungen, negative Gefühle zu entwickeln. Sie sehen sich also emotional als die Opfer. Vgl. auch Haubl/Caysa (2007: 96): „Der Sündenbock ist selbst Schuld daran, dass man ihn hasst. Das Opfer ist [...] immer für alles verantwortlich und es ist auch verantwortlich für die Anschuldigungen, die ihm zu teil werden, egal, ob sie nun den Tatsachen entsprechen oder nicht. Der Hass erschafft erst sein Gegenüber als Feind [...].“ 


\section{Neid}

Das Gefühl des Neides wird im Vergleich zu Hass, Wut, Zorn und Abscheu/Ekel sehr selten ausgedrückt (in den Tausenden von E-Mails wird das Lexem Neid lediglich zwölfmal benutzt und kommt dabei nur bei rechtskonservativen bzw. rechtsextremen Autoren vor). Auch implizite Gefühlsausdrücke zu Neid wie in (33) sind nicht oft zu konstatieren.

„Sie sprechen immer von Antisemitismus in Deutschland. Welche riesen Vorteile beziehen Sie daraus - Sie brauchen nur den Finger zu heben, schon werden Sie vom Deutschen Staat reichlich mit Geld beschenkt.“ [ZJD_27.07.2006_ano_008]

Signifikant ist, dass bei diesen Texten Neidgefühle immer gekoppelt an die Stereotype von JUDEN ALS AUSNUTZER/PARASITEN/MÄCHTIGE NUTZNIESSER vorkommen:

(34) „Juden waren es auch auch, die die perfiden Strategien der Bereicherung, die sich in unserer Welt durch den Einzug des Kapitalismus breit gemacht haben, als erstes schamlos ausgenutzt und über die Welt verbreitet haben. So kommt es nicht von ungefähr, daß viele von ihnen einen großen Reichtum und auch viel Einfluß in der Wirtschaft erlangt haben und sie sich für das Wohnen auch mit die schönsten Plätze unter den Nagel gerissen haben (ich erinnere in diesem Zusammenhang nur an die Liebermannvilla in Berlin Wannsee).“ [ZJD_30.11.2006_Gel_001]

(35) „Das ist eine Ausnutzung des Holocaust-Bonus, den Sie mißbrauchen.“ [ZJD_31.08.2006_Ros_001]

Die insgesamt aber signifikant schwache Ausprägung von emotionsbezeichnenden und -ausdrückenden Mitteln zu Neid und Missgunst ist ein klarer Indikator dafür, dass dieses Gefühl (oder eine „Mischung destruktiver Neidgefühle“, Hegener 2006: 19f.) keine wesentliche Rolle beim aktuellen Antisemitismus spielt. Und auch aus historischer Perspektive führt ein Ansatz, wie er z. B. von Aly (2011) vorgelegt wurde, der versucht, den eliminatorischen Judenhass mit seiner pseudo-religiös und ideologisch fundierten Erlösungskomponente eher auf materielle Spannungen und ökonomische Interessen zurückzuführen, in eine Sackgasse (s. auch Kap. 4.3). 
Das Emotionspotenzial der Texte gibt insgesamt nicht nur Einblick in die individuellen Gefühlsmanifestationen der Schreiber, sondern verrät viel über kollektive Gefühle, die konstitutiv für Judenfeindschaft sind.

\title{
9.3 Die obsessive Dimension
}

\subsubsection{Grausamkeit und Gefühlskälte}

Aus den Gefühlen von Hass, Ärger, Zorn und Wut, die alle zu den destruktiven Emotionen zählen und der Dimension menschlicher Aggressivität zuzuordnen sind, ergibt sich zum einen eine mitleidslose Gefühlskälte, zum anderen eine grausame Brutalität gegenüber den als FEINDE, STÖRENFRIEDE, UNMENSCHEN konzeptualisierten Juden. Die aggressive Feindseligkeit artikuliert sich in Äußerungen wie (36) und (37):

„Hoffentlich sterben noch viele Judenkinder-nur so lernt ihr Taeter.“ [ZJD_27.12.2008_Did_001]

\begin{abstract}
„Und solange Scharon den Palästinensern das Recht auf einen eigenen Staat vorenthält, soll auch Israel keine Ruhe finden und in Angst vor den nächsten Attentaten leben.“ [ZJD_24.06.2002_Mau_001]
\end{abstract}

Eine auffällige Gefühlskälte gekoppelt an aggressive und für eine zivilisierte Wertegemeinschaft atavistisch anmutende Verwünschungen gegenüber Juden und Israelis drückt sich in vielen der Schreiben aus. So bekennt ein Herr aus BadenWürttemberg per Fax:

„Am jüdischen Symbol dem Stern klebt Blut und ich habe kein Mitleid mit den Opfern in Israel.“ [IBD_12.08.2006_Sch_003]

Den emotionalen Selbstdarstellungen, die alle Anzeichen intensiv erlebter Zustände und Prozesse sind, steht eine ostentative Gleichgültigkeit gegenüber, wenn es um jüdische und/oder israelische Opfer ${ }^{11}$ geht, die oft untrennbar gekop-

11 Im konzeptuellen Glaubenssystem der „antisemitischen Ethik“ gibt es ein „just-world thinking, which is the tendency to believe that people who suffer, especially those already devalued, must deserve their suffering as a result of their deeds or their characters; and scapegoating, or blaming others for one's problems " (Staub 51995: 17). Vgl. auch Haubl/Caysa (2007: 33 ff.): „Ihre Bekämpfung und Ausrottung geschieht ohne jegliches Schuldgefühl.“ 
pelt ist an eine feindselige Aggression, die darin besteht, Juden und/oder Israelis intentional Schmerz, Verletzungen oder den Tod zu wünschen (s. hierzu ausführlich Kap. 10).

„Betreff: Jüdische Verbrechen in Hebron. Jemand möge ihr Haus stürmen, Sie auf die Straße setzen etc.“[ZJD_05.12.2008_ano_001]

„Ich hoffe und wünsche, dass ... viele, viele Israeli sterben und ihre Familien leiden müssen.“ [IBD_22.03.2004_Sei_001]

So artikuliert auch der Verfasser von (41) mit großer Emphase seine Gewaltphantasien:

„Ich bete zu Gott-den Juden nicht zu kennen scheinen-das die gleiche Anzahl Israelis jämmerlich zu Grunde gehen wie es die Kinder und Frauen der Palestinänser taten die ein Recht auf ihr Land haben das ihnen teilweise von Kriegsverbrechern gestohlen wird“ [IBD_20.02.2009_Wei_001]

Die Schreiber werfen dabei in ihren Texten Juden und Israelis mehrheitlich „moralisches Versagen“, „unglaubliche Grausamkeit“ und „Mitleidlosigkeit“vor. Sie verbalisieren mit solchen Fremdzuschreibungen aber exakt die Gefühlszustände, in denen sie sich selbst befinden. Über die Sprache projizieren sie so (erstaunlich präzise und sehr genau benannt) ihre eigenen emotionalen Defizite ${ }^{12}$ auf das mentale Konstrukt JUDE.

Solche verbalen Gewaltakte mit Visionen eines derart destruktiven Ausmaßes können nur artikuliert werden, wenn keinerlei Empathie für die Menschen vorhanden ist, denen man dies wünscht. Empathie ist die Fähigkeit, sich selbst in die Lage eines anderen Menschen $\mathrm{zu}$ versetzen und so $\mathrm{zu}$ empfinden wie

12 Solche Äußerungen sind keineswegs auf das E-Mail-Korpus beschränkt. In zahlreichen Internet-Kommentaren finden sich, teils explizit, teils implizit, Aussagen, in denen die Sprachproduzenten Gefühle wie Mitleid für Juden oder Israelis verweigern. Im öffentlich einzusehenden Kommentarbereich von ARD und ZDF z. B. waren im März 2011 dutzende Einträge der folgenden Art zu lesen: „Wer Gewalt sät, erntet Gewalt. Das müßte den bibelfesten Juden doch wohl bekannt sein.“ [12.03.2011_17:23_Raimon] oder „Diese Siedlungen waren sind und bleiben ILLEGAL. Warum nur wird das in diesem Artikel mit keinem Wort erwähnt??! [...] Da kann man den israelischen Siedlern nur jeden gesunden Menschenverstand absprechen, da sie ihre Kinder in eine sehr gefährliche Lage bringen [...].“ [12.03.2011_20:35_Maysoon] Anlass war ein Artikel zu einem besonders brutalen Mord an einer israelischen Familie in Itamar, die im Schlaf erstochen worden war, inklusive drei kleiner Kinder, das jüngste Mädchen drei Monate alt, auch ihre Kehle wurde durchgeschnitten. 
dieser. Ein Mensch, der für andere Empathie fühlt, ist nicht in der Lage, ihnen gleichzeitig Schmerz und Leid zu wünschen. Denn wenn eine Identifikationsbasis gegeben wäre, könnten solche grausamen Verwünschungen kaum artikuliert werden. Da die feindselige und entmenschlichende Konzeptualisierung von JUDE jedoch eine Einfühlung, Annäherung oder Identifikation verhindert, ${ }^{13}$ kann auf der Basis dieser absoluten Entwertung nur emotional radikal gefühlt werden.

Die Empathieverweigerung gegenüber Juden und Israelis steht in auffälligem Kontrast zu den intensiven Gefühlsbekundungen, die sich auf die Schreiber selbst, die „intensiv leiden“, sich „so sehr sorgen“ und „in empörter Rage“ sind, aber auch (projiziert) auf die angeblichen Opfer der „Täter-Juden“ beziehen (s. hierzu auch Schwarz-Friesel 2010a: 47 und 2012b).

„Ich verspuere nur Ekel, Abscheu und bin nach ueber 30 Jahren, die ich mich fuer Israel eingesetzt habe, nicht laenger bereit, dieses Land und seine diskriminierende Politik, die ein ganzes Volk seit Jahrzehnten unter die Knute zwingt und nicht menschenwuerdig leben laesst, zu unterstuetzen.......es reicht!!“ [ZJD_26.07.2006_ano_005]

(43) „Ich bin zu tiefst erschüttert über die Verlogenheit der Zionisten.“ [ZJD_26.07.2006_Koe_002]

Sie sind „voller Mitgefühl“ und „ohnmächtiger Wut“, es „,bricht“ihnen „das Herz“, wenn sie an „das Elend der Palästinenser“ denken und sie sind „fassungslos angesichts der Herzlosigkeit von Juden“, wie der Verfasser von (44), ein Arzt:

(44) „Mein Mitleid und meine Solidarität gilt dem palästimensischen Volk . Übrigens: viele meiner Patienten denken und fühlen ähnlich.“ [ZJD_Gaza2009_160/816_Dös_001]

So befindet etwa ein Ingenieur aus Nürnberg in seiner E-Mail an den Zentralrat:

„Ich selbst empfinde gleichzeitig Trauer und ohnmächtige Wut ob ihrer Blindheit. Ihr Gruß heißt "Friede”. Warum leben Sie ihn nicht? Shalom!“ [ZJD_03.08.2006_Stu_001]

13 „Empathie lässt sich dem Hassobjekt umso leichter verweigern, wenn es mit allen sprachlichen und bildlichen Mitteln als das nicht einfühlbare Andere dargestellt wird“ (Haubl/ Caysa 2007: 46). Wir gehen davon aus, dass die verbale Dehumanisierung Resultat der mentalevaluativen Entmenschlichung ist. 
Ein Dipl.-Ökonom aus Bochum artikuliert seine Gefühle ebenfalls sehr drastisch:

(46) „Es ekelt mich an, was Israel z.Zt. im Libanon treibt! Ich würde mich gern vor Ihrer Botschaft erbrechen, um zu unterstreichen, was ich von Ihrer Agressionspolitik halte...“ [IBD_11.08.2006_Loe_001]

Offenkundig gibt es bei diesen Schreibern zweierlei Maß bei der Empfindung bzw. Bekundung von Empathie: zum einen Empathieverweigerung in Bezug auf Juden und Israelis, zum anderen ostentative und intensive Empathie für die „Opfer des zionistisch-jüdischen Terrors“. Diese emphatisch ausgedrückte Identifikation transferiert aber wohl nur das eigene Selbstmitleid der Verfasser auf eine moralisch wie gesellschaftlich akzeptable Ebene, nämlich die des Mitgefühls. Als nach außen projiziertes „Mitleid“ deklariert werden die destruktiven Gefühle von Hass und Wut legitimiert, zugleich wird das Selbstmitleid extern kanalisiert. So dient die Identifikation mit den „Opfern der Juden“ am Ende der Empathie mit sich selbst. Und sie hilft der Verdrängung der unter Umständen unangenehmen und emotional belastenden Selbsterkenntnis, dass die intensiven Gefühle einer tiefen mentalen Judenfeindschaft entspringen.

\subsection{2 Überdrussmentalität und Empathieverweigerung}

Als eine Form der emotionalen Grausamkeit lässt sich auch die Empathieverweigerung vieler Schreiber charakterisieren, wenn sie Juden vorwerfen, sie würden „zu viel über den Holocaust reden“" (was sich auch in diversen Umfragen zeigt) und fordern „endlich mit der ewigen Erinnerungs- und Mitleidstour“ aufzuhören.

(47) „Halten Sie bitte endlich den Mund. Der 2. Weltkrieg ist seit über 60 jahren vorbei.“[ZJD_19.04.2007_Mue_001]

Die Schreiber finden es mittels drastischer Emotionsausdrücke „zum Kotzen“, „erbärmlich“ und „als widerwärtige Mitleidsschiene“, dass Juden mahnend an die Gräueltaten in der NS-Zeit erinnern und der Opfer gedenken wollen.

(48) „ich kann Holocaust nicht mehr hören. Und den Zeigefinger vom Zentralrat der Juden nicht mehr sehen. Es ist genug!“ [ZJD_13.06.2002_Mau_001]

(49) „Ihr hängt mir einfach mit Eurem ewigen drögen Gejammere zum Halse heraus - und der Mehrheit der Bürger auch!“ [ZJD_23.11.2007_ano_001] 
Das Zurückweisen der Erinnerungskultur ist gekoppelt an die Verweigerung eines emphatischen Gefühls: Keinerlei Verständnis wird für das Bedürfnis der Opfer(nachkommen) gezeigt, die Erinnerung an den Holocaust wachzuhalten.

(50) „Ich kann es nicht mehr ertragen, daß die Herren Spiegel und Friedmann ständig mit erhobenen Zeigefinger vor den Deutschen herumfuchteln und sich als Moralapostel aufspielen.“ [ZJD_08.06.2002_Her_001]

Im Gegenteil: Dieses Bedürfnis wird den Betroffenen als „Ruhestörung“ und „Grund für Antisemitismus“vorgeworfen (s. Kap. 5).
„Aber wir müssen ja wieder an unsere ewige Kollektivschuld erinnert werden. Immer und immer wieder - bis es auch dem letzten zum Hals raushängt und sich antisemitische Gefühle entwickeln.“
[ZJD_01.05.2006_ano_001]

Die Schlussstrich-Forderung stützt sich dabei (auch bei Verfassern aus der Mitte) auf das Argument, lange genug sei der Erinnerungs- und Gedenkpflicht genüge getan und es seien hinreichend finanzielle Reparationen geleistet worden (s. hierzu auch Bsp. (164) in Kap. 5.2).

Funktional gekoppelt ist die Erinnerungsabwehr oft auch an Schuld- und Verantwortungsabwehr (s. hierzu bereits Adorno [1962] 1971: 115; s. auch Rensmann 2004 und Bergmann ${ }^{32006)}$ und die Forderung, einen „Schlussstrich unter die Vergangenheit zu ziehen" und sie ruhen zu lassen (oft in Verbindung mit TäterOpfer-Umkehrungen):

$$
\begin{aligned}
& \text { „hören sie auf mir als deutschen Staatsbürger der } 1961 \text { geboren wurde } \\
& \text { eine kollektive Mitschuld zu verkaufen am Holocaust, denn es ist eine } \\
& \text { Frechheit mich verantwortlich zu machen an dem was ich gar nicht } \\
& \text { beinflussen konnte! Desweiteren schauen sie selbst mal in den spiegel } \\
& \text { welche Nazi-Methoden der staat israel angewerndet hat und noch heute } \\
& \text { ausübt!“[IBD_19.07.2011_Fre_001] }
\end{aligned}
$$

Verbunden mit dieser Schlussstrich-Forderung ist das Gefühl, durch die moralischen Appelle von Juden am Pranger zu stehen, und damit keinen Nationalstolz entwickeln zu dürfen.

(53) „Wir brauchen nach meiner Meinung keine ständigen Ermahnungen der Marke Knobloch oder Friedmann mehr -- ständige Veranstaltungen bei denen unsere Politiker zu Kreuze kriechen müssen und die dann noch 
den Steuerzahler viel Geld kosten. Wer heute noch ständig Angst vor den neuen deutschen hat der sollte sich wirklich überlegen ob Deutschland dass richtige Land für ihn ist ,es steht jedem frei auszureisen und uns mit seiner ständigen Erinnerungs und Mitleidstour verschonen ( Wir können und wollen es ncht mehr hören ).“[ZJD_10.11.2008_Hub_001]

Die Überdrussmentalität spiegelt sich in der Weigerung wider, Gefühle von Scham, Entsetzen und Trauer zu empfinden (s. hierzu maßgeblich Mitscherlich/ Mitscherlich 1967). Auch von den Opfern und ihren Angehörigen wird verlangt, nicht länger die Öffentlichkeit mit ihrem Schmerz zu behelligen. Oft schwingt dabei der stereotype Vorwurf mit, Juden in Deutschland würden in mahnender Funktion auftreten, um sich Vorteile zu verschaffen, so wie in der E-Mail eines Herrn aus dem Sauerland:
„Sie haben sich mit der 'deutschen Schuld' schon bei so vielen deutschen Politikern (z. B. J. Rau) ihre Vorteile verschafft, so daß wir Ihre Moralpre- digten nicht mehr hören können.“ [ZJD_27.07.2006_Bar_001]

Dass angesichts des Ausmaßes von Leid und Zerstörung und präzedenzloser Inhumanität für die traumatisierten Opfer(familien) und deren Nachkommen die Erinnerung notwendig und unverzichtbar ist und auch jede zivilisierte und mit der eigenen Geschichte verantwortlich umgehende Gesellschaft auf eine Erinnerungskultur, die dem größten Zivilisationsbruch in der Menschheitsgeschichte gedenkt, nicht verzichten kann und darf, wird (auch von vielen Akademikern) ${ }^{14}$ nicht in Betracht gezogen. Die Geschehnisse in der NS-Zeit werden emotional nicht als moralische Last, die innerlich zu existenzieller Trauer, Mitgefühl und Reflexion verpflichtet, sondern als ein Lästig-Sein empfunden, das von den Juden oktroyiert wird und das es abzuschütteln gilt. Ein Widerspruch wird dabei offenkundig: Die Erinnerungskultur wird einerseits als inflationär und als lästige Bürde empfunden, andererseits zeigt die massive Verwendung der VerbalAntisemitismen der Verfasser, dass eine tiefgehende Aufarbeitung der Ursachen tradierter Judenfeindschaft dringend nötig wäre. Zum einen empfinden also viele Deutsche ein „Zuviel“ an Erinnerungskultur und beklagen die „Überflu-

\footnotetext{
14 Entsprechend werden auch Ergebnisse der Antisemitismusforschung, die zeigen, wie präsent judenfeindliches Gedankengut in der Mitte der Gesellschaft ist, vehement geleugnet und marginalisiert. Die Nie-Wieder-Mentalität in Bezug auf den Holocaust beschränkt sich bei vielen Menschen auf wenige Gedenktage im Jahr. Zur reinen Floskel erstarrt sind Phrasen wie Gedenken an die Opfer und Wir sind uns unserer Verantwortung bewusst. Eine kritische Auseinandersetzung mit der problematischen Gegenwart wird so verhindert.
} 
tung“ durch Studien zur NS-Zeit, zum anderen wissen heute, wie Umfragen in den letzten Jahren gezeigt haben, viele junge Deutsche nicht einmal mehr, was Auschwitz bedeutet und wofür es steht. ${ }^{15}$ Dieser Widerspruch offenbart die Diskrepanz zwischen subjektiver, gefühlter Einschätzung und objektiver Situation. Im Bewusstsein vieler (auch hoch gebildeter) Deutscher - dies zeigen sowohl die Korpus-Texte als auch diverse Umfragen der letzten Jahre - ist die Konzeptualisierung vorhanden, nach 1945 habe es eine andauernde, lückenlose und intensive Vergangenheitsbewältigung gegeben, die alle erreicht habe, die umfassend aufgeklärt und die Bürger kollektiv sensibilisiert habe. Als sei kontinuierlich auf allen gesellschaftlichen Ebenen aufgearbeitet, erinnert und ermahnt worden. Dass dies aber imaginiert ist (und nach dem Zusammenbruch des NS-Regimes über 20 Jahre lang die Gräueltaten zunächst weitgehend ignoriert und relativiert wurden und so die Chance verpasst wurde, den Holocaust als Zäsur im kollektiven Bewusstsein zu verankern), haben Studien zum Nachkriegsdiskurs deutlich gezeigt (s. Kap.4.4). Die Verfasser selbst führen diese Einschätzung zudem ad absurdum, indem sie in ihren stereotyp- und ressentimentbelasteten Schreiben erkennen lassen, dass ihnen Toleranz, Verständnis und Empathie fehlen, sie also keineswegs Lehren aus der (angeblich so überdimensional präsenten bzw. präsentierten) Geschichte gezogen haben.

\subsection{Wider die Vernunft: Zur Dominanz der irrationalen Dimension in antisemitischen Texten}

\subsubsection{Trugschlüsse und selbsterfüllende Prophezeiungen}

\footnotetext{
„How is it that men believe of the Jews what common sense would forbid them to believe of anything else? Why? There can be but one answer: people believe such things because they want to believe them.“ (Trachtenberg 1943: 1f.)
}

Der Einfluss von negativen Emotionen (als irrationale Störfaktoren, die Vernunft und Logik zuwiderlaufen) zeigt sich auch sehr deutlich bei den widersinnigen

15 S. hierzu z. B. die Forsa-Umfrage für den Stern zum Holocaustgedenktag im Januar 2012. 21 Prozent der 18- bis 29-jährigen Befragten wussten nicht, wofür das Wort Auschwitz steht; 31 Prozent aller Befragten wussten nicht, in welchem Land Auschwitz liegt. 40 Prozent aller Befragten wollen aber einen Schlussstrich unter die Vergangenheit ziehen und 65 Prozent aller Befragten gaben an, die Deutschen hätten aufgrund ihrer Geschichte keine besondere Verantwortung gegenüber anderen Völkern. In der ADL-Umfrage von 2009 kreuzten 44 Prozent der Befragten in Deutschland die Aussage an „Juden beziehen sich zu oft auf den Holocaust“. 
und paradoxen Argumentationsstrukturen sowie der semantisch-konzeptuellen Inkohärenz der Texte.

Kaum eine der Zusendungen an den Zentralrat der Juden oder die Israelische Botschaft in Deutschland kommt ohne Begründungen oder Argumentationen ${ }^{16}$ aus (s. hierzu Kap.11). Von reinen Solidaritätsbekundungen und einigen sehr kurzen Hassbotschaften abgesehen, besteht bei den Produzenten der Texte ein großes Bedürfnis, die eigene Stellungnahme und die oft daran anschließenden Forderungen argumentativ zu untermauern und durch die Angabe von Begründungszusammenhängen rational zu stützen. Diese Form der Obsessivität zeigt sich in zahlreichen „Beweisführungen“ der Verfasser (deren Umfang teilweise den von Seminararbeiten - inklusive Anhängen, Literaturzitaten und Fußnoten - hat):

„Sehr geehrter Herr Spiegel, ich lege Ihnen eine Übersicht über geschichtliche Fakten bei und ich bitte Sie, diese Fakten genau zu beachten.“ [ZJD_08.04.2002_Lud_001]

Dabei kommt es jedoch (vor allem aufgrund der hohen emotionalen Involviertheit) häufig zu Trug- und Fehlschlüssen innerhalb der Argumentationen. ${ }^{17}$ Das Resultat sind pseudo-rationale Argumentationsketten, die auf der Oberfläche den Regeln des Verstandes zu folgen scheinen, tatsächlich aber semantisch-konzeptuelle Fehler beinhalten.

Solche ungültigen und den Prinzipien der Vernunft zuwiderlaufenden Argumente werden als Fallacien ${ }^{18}$ bzw. Trugschlüsse bezeichnet. Sie entstehen durch

16 Argumentieren meint den Versuch eines Sprachproduzenten, den begründungsbedürftigen Geltungsanspruch einer Äußerung durch Berufung auf die unterstellte Gültigkeit einer anderen Äußerung einzulösen (s. hierzu u. a. Kopperschmidt 1989). Argumentationen zielen somit darauf, dass sich der Rezipient der Sichtweise des Produzenten anpasst, und zwar auf dem Weg über kognitive Prozesse (vgl. Sornig 1986: 251). Sie beruhen auf (alltags)logischen Schlüssen, also auf einer expliziten oder impliziten regelhaften Beziehung (s. Toulmin 1958).

17 Adorno ([1946] 2002: 153) hat bereits auf einige Eigenarten dieser Pseudo-Argumentation hingewiesen: „Sie [= die faschistische Propaganda, d. Verf.] folgt keiner diskursiven Logik, sondern läßt sich - besonders bei rhetorischen Darbietungen - eher als eine Art organisierter Gedankenflucht kennzeichnen. Die Relation zwischen Prämissen und Schlußfolgerungen wird durch eine Verknüpfung von Gedanken ersetzt, die auf bloßer Ähnlichkeit beruht und bei der oft ein und dasselbe charakteristische Wort in zwei Behauptungen figuriert, die in keinem Zusammenhang stehen, aber durch dieses Wort assoziativ verbunden werden. Dieses Verfahren entzieht sich nicht nur den Kontrollmechanismen einer rationalen Prüfung, sondern macht es dem Zuhörer leichter, zu 'folgen'.“

18 Vgl. Löhner (1996), van Eemeren (2001). In der (historischen) Rhetorik unterscheidet man zwischen einem Trugschluss, d. h. einem formal unrichtigen Schluss, der mit der Absicht 
den fehlerhaften inneren Aufbau eines Arguments, die Ziehung falscher Schlüsse aus den Prämissen eines Arguments oder durch eine anfechtbare Stützung bzw. fehlende Faktizität dieser Prämissen. In den judenfeindlichen Schreiben basieren Fallacien quantitativ am häufigsten auf dem Rückgriff auf falsche Prämissen, der Ausblendung von Fakten sowie dem Heranziehen unzulässiger stereotyper Topoi durch die Textproduzenten. Die starren kognitiven Stereotyprepräsentationen und die zum Teil starke Erregtheit bringen die Verfasser dazu, die Gesetze der logischen Argumentation außer Acht zu lassen bzw. zu verletzen, was ein typisches Kennzeichen antisemitischer Argumentation ist.

\title{
Fallacien aufgrund falscher Prämissen
}

Oft entstehen Trugschlüsse dadurch, dass die Textproduzenten von falschen Prämissen ausgehen, d. h. falsche Thesen bzw. vermeintliche Fakten als Ausgangsbasis für ihre Argumentation nutzen.

\begin{abstract}
„Unser deutsches Volk kämpft ums finanzielle Überleben, weil es von den Politikern verursachte Abgaben mehr und mehr stranguliert wird. Es wird in Berlin ein Holocaustdenkmal für 60 Millionen errichtet. Das ist ein Verbrechen am deutschen Volk.“[ZJD_04.06.2002_Ric_001]
\end{abstract}

Der Verfasser aus Monheim geht von der Prämisse aus, dass sich das deutsche Volk bzw. der deutsche Staat aufgrund von Staatsschulden in einem Kampf um das „finanzielle Überleben“ befinde (was eine de-realisierende Übertreibung ist). Da die erste Prämisse falsch ist, ist auch die Konklusion, das Berliner Holocaustdenkmal sei ein Verbrechen, falsch. Diesen argumentativen Trugschlussprozess kann man gut mit dem Argumentmodell nach Toulmin (1958) darstellen. Dieses baut auf dem klassischen Syllogismus auf, also einer Form von Argumenten, bei denen sich aus zwei Prämissen eine Konklusion ergibt. Toulmin erweitert dieses Modell durch eine Stütze, die erklärt, aus welchem argumentatorischen Feld heraus eine der beiden Prämissen generiert wird.

gemacht wird, andere zu täuschen, und einem Fehlschluss (Paralogismus), d. h. einem falschen Schluss, bei dem sich der Sprachproduzent unbeabsichtigt selbst täuscht. Die in den Korpustexten vielfach zu beobachtenden Fehlschlüsse sind mutmaßlich nicht-intentional gezogen worden. Die Verfasser sind von der Richtigkeit ihrer Aussagen und Urteile überzeugt, denn sie stellen das Fundament ihrer judenfeindlichen Einstellung dar. Letztlich lässt sich aber die Frage, inwieweit die Schreiber sich der Falschheit der verwendeten Thesen bewusst sind oder nicht, durch eine reine Korpusanalyse nicht sicher beantworten. Die Sprechhandlungen könnten prinzipiell auch den Status Lüge haben. 
Eine Aufschlüsselung von Beispiel (56) nach diesem Modell sieht dann wie folgt aus:

These (1. Prämisse)

Konklusion

\begin{tabular}{|c|}
\hline $\begin{array}{c}\text { das dt. Volk kämpft um } \\
\text { sein finanzielles } \\
\text { Überleben }\end{array}$ \\
\hline
\end{tabular}

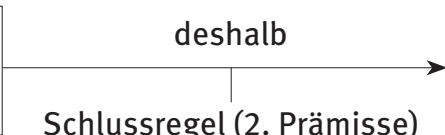

das (teure) Holocaustdenkmal war ein Verbrechen

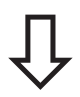

1. Prämisse falsch
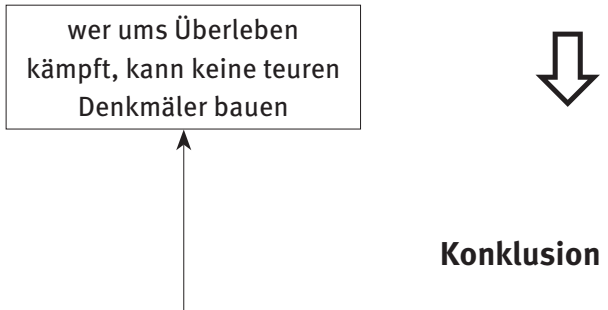

Stütze

Auffällig ist bei vielen dieser Fallacien, dass häufig entweder nicht relevante Fakten, sondern irgendwelche (zum Teil beliebigen) oder erkennbar und nachweislich falsche Fakten angeführt werden, um die eigene Haltung oder Einstellung zu begründen. Diese werden dann als scheinbar aussagekräftig und sachbezogen eingeflochten (so hat die enorme Staatsverschuldung noch keine Regierung davon abgehalten, unnötige Investitionen zu tätigen). Man kann davon ausgehen, dass der Bau des Holocaustmahnmals für den Verfasser von (56) ein Ärgernis bzw. eine Provokation darstellt, die seiner auf Ablehnung der Erinnerungskultur basierenden Einstellung zuwiderläuft. Um seine Missbilligung nicht bloß als individuelle Meinung kundtun zu müssen, greift er daher auf scheinbar plausible, für die Allgemeinheit akzeptable und nachvollziehbare Gegenargumente zurück, die er im Themenspektrum der Staatsfinanzen bzw. Haushaltskonsolidierung findet.

„Palästinensische Kinder werden von den Israelis immer brutaler behandelt und ermordet. Die Zahlen steigen ständig. Die Welt ist ohne Israelis besser dran. Also ist das Fazit die Israelis auszurotten die einzige Lösung.“ [IBD_15.10.2007_Dro_001]

Aus der Prämisse, die auf der de-realisierten Weltsicht des Verfassers von (57) konstruiert wurde, die durch den scheinbar faktisch basierten, aber ebenfalls imaginierten Bezug auf steigende Zahlen gestützt wird, ergibt sich aus der judeo- 
phoben Sicht des Verfassers zwangläufig nur eine Konklusion: die Notwendigkeit der Eliminierung aller Israelis. Solche Konklusionen entstehen nach dem Prinzip der selbsterfüllenden Prophezeiungen: ${ }^{19}$ Sie existieren als unerschütterliche Glaubenssätze (z. B. 'Alle Israelis sind jüdische (Kinder)Mörder und Verbrecher') bereits im Kopf der Sprachproduzenten, werden aber als Fakten ausgegeben, die der Realität entsprechen und durch selbst erfüllende, den Glaubenssatz bestätigende „Argumentationen“ etabliert. Bezogen auf das Phänomen der Judenfeindschaft lässt sich dies folgendermaßen beschreiben: Im mentalen Modell von Antisemiten sind die Konzepte zu Juden an bestimmte stereotype Inhalte gekoppelt, die Voraussagen zu deren Verhalten beinhalten, z. B. 'Juden sind grausam und hinterlistig'. Alles, was Juden tun, ist per definitionem ${ }^{20}$ schlecht und sämtliche pseudo-kausalen Urteile und Motive, die genannt werden, sind Rationalisierungen dieses Ressentiments. Der Generalverdacht gegen Juden, ein Konglomerat aus Argwohn, Misstrauen, Unkenntnis und fixen Stereotypen, findet hier seinen verbalen Ausdruck. Jede Gelegenheit und jede Information, und sei sie auch noch so unplausibel und vage, wird benutzt, um diesen Generalverdacht zu stützen und $\mathrm{zu}$ bestätigen.

Israelische Militäraktionen werden nach diesem Glaubenssystem entsprechend als grausam und hinterlistig bewertet. Eine andere Lesart wird kategorisch ausgeschlossen bzw. nicht einmal in Erwägung gezogen. So produzieren die Verfasser selbst systematisch genau die Bestätigungen, die sie erwarten. Formal wird Konsistenz etabliert, die aber eine konzeptuelle Scheinkonsistenz ist (s. hierzu auch Hacker 1990: 119).

\section{Fallacien durch Stereotype}

In zahlreichen Texten kommt es zur Simulierung von (scheinbarer) Plausibilität im Sinne von kausal bedingten Begründungszusammenhängen, indem antijüdische Stereotype als Stütze für Prämissen genutzt werden. Diese Stützen sind jedoch per se falsch, weil sie auf ungerechtfertigten Pauschalgeneralisierungen oder Fiktionen beruhen (vgl. Kap. 5).

19 Selbsterfüllende Prophezeiungen (engl. self-fulfilling prophecies) nennt man Vorhersagen, die sich erfüllen, weil diejenigen, die an diese Prophezeiung glauben, sich (oft unbewusst) aufgrund der Prophezeiung so verhalten, dass sie sich erfüllen bzw. bei anderen gerade das Verhalten entweder provozieren (oder als solches interpretieren), dass sie vorhergesagt haben. „The process by which one person's expectations about another become reality by eliciting behaviors that confirm the expectations" (Smith/Mackie: ${ }^{2} 2000$ : 94).

20 S. hierzu auch Sartre (1948: 10): „Nicht die Erfahrung schafft den Begriff des Juden, sondern das Vorurteil fälscht die Erfahrung.“ 


\begin{abstract}
„Die Deutschen wollen nicht mehr arbeiten, weil sie so viele Steuern und Renten zahlen müssen. Die Renten werden gekürzt und die Rentenerhöhung wird jedes Jahr niedriger. Wenn so weiter geht, haben die Ausländer in Deutschland das Sagen. Bei Handel und Industrie sind die Juden und Amerikaner die größten Kriegstreiber und die Blutrünstigsten der Welt.“[ZJD_13.09.2002_ano_004]
\end{abstract}

So entsteht in Beispiel (58) Kohärenz, d.h. inhaltlicher Zusammenhang innerhalb des Textes, allein durch den Prozess einer stereotypbasierten Inferenzziehung. Nur wenn man die Schlussfolgerung zieht, dass sich die Konzepte AUSLÄNDER und JUDEN des Textproduzenten stark überschneiden (dass er also deutsche Juden als NICHT-DEUTSCH konzeptualisiert und damit das antisemitische Stereotyp von JUDEN ALS FREMDE verinnerlicht hat) und mit dem Konzept AUSLÄNDER den Informationsknoten SIND VERANTWORTLICH FÜR RENTENKÜRZUNGEN verbindet, ergibt die Äußerung einen kohärenten Sinnzusammenhang.

\title{
Heranziehen unzulässiger Topoi
}

Topoi, konzeptuelle Kategorien mit Themenschwerpunkten und zum Teil hoher bildlicher Vorstellungskomponente, sind mentale Quellen für Argumente. Man kann mit ihrer Hilfe sowohl Pro- als auch Kontra-Argumente in Bezug auf eine bestimmte (Streit)Frage generieren, jedoch nicht mit ihnen selbst argumentieren. Beispiele für solche Topoi sind Freiheit, Ökonomie oder auch Vergleichsschlüsse.

$$
\begin{aligned}
& \text { „Der Anlaß zu meinem Schreiben ist das menschenverachtende Vor- } \\
& \text { gehen der israelischen Streitkräfte im Gaza und vor allem im Libanon. } \\
& \text { [...] Es ist müßig, danach zu fragen, wer den ersten Stein warf, die Jahr- } \\
& \text { tausende der jüdischen Geschichte sind voll von dem gewaltsamen, } \\
& \text { blutigen Vorgehen der Hebräer gegen ihre Nachbarn oder wenn es sein } \\
& \text { musste auch gegen unbotmäßige eigene Stämme. So ist das Pessach-Fest } \\
& \text { das Gedenken an ein furchtbares Massaker, bei dem alle erstgebore- } \\
& \text { nen Ägypter erschlagen wurden, nicht von Jahwe, sondern von seinem } \\
& \text { gewaltbereiten Volk. [..., Bibelzitat] Bei der Eroberung von Jericho wurde } \\
& \text { alles Lebende umgebracht, Männer, Frauen, Kinder und auch Tiere, wie } \\
& \text { in der Bibel nachzulesen ist. Dieser Faden der Gewalt zieht sich durch die } \\
& \text { Jahrtausende und bis heute nicht abgerissen.“[ZJD_29.07.2006_Gru_001] }
\end{aligned}
$$

In Beispiel (59) werden mithilfe des Topos 'Religion(sschriften)' bzw. des Vergleichsschlusses 'wie in der Bibel, so in der Gegenwart' Argumente gegen das 
Vorgehen des israelischen Militärs, die aber kollektiv gegen alle Juden gerichtet sind, produziert. Dies wäre in einem religiösen Disput zumindest rhetorisch legitim, obwohl diese Art der Argumentation moralisch bedenklich und logisch falsch ist; der Textproduzent befindet sich jedoch nicht in einer solchen kommunikativen Situation. Er schreibt an den Zentralrat der Juden in Deutschland und argumentiert in einem politisch-militärischen Diskurs der Gegenwart. In diesem Zusammenhang ist sein vergangenheitsbezogener und religiöser Topos nicht zulässig und bedient zudem das judenfeindliche Klischee vom ewigen Juden als Unruhestifter. Das abschließende Argument, Gewaltanwendung sei dem Judentum seit Jahrtausenden inhärent, und die daraus zu inferierende Konklusion, dass das Verhalten israelischer Streitkräfte genau dieser blutigen Tradition entspreche, ergibt sich also ausschließlich aus dem stereotypen mentalen Schema JUDEN, das im Kopf des Schreibers gespeichert ist.

\section{Ignorierung, Ausblendung und Umdeutung von Fakten}

In Beispiel (60) äußert ein Akademiker aus München ebenfalls Argumente, die zu einer unzulässigen Schlussfolgerung führen, da er relevante historische und aktuelle Fakten außer Acht lässt. Er argumentiert rein militärstrategisch und vernachlässigt dabei zum einen, dass ein Staat und seine Existenzsicherung von mehr als seinem Militäretat abhängen, zum anderen, dass Israel bereits vielfach in Kriege verwickelt war, die von den umgebenden arabischen Staaten initiiert wurden. Ignoriert wird auch die feindselige, Gewalt androhende Rhetorik gegenüber Israel von vielen Staaten seiner Umgebung sowie die terroristischen Anschläge auf seine Bevölkerung innerhalb des Landes. Die Reduktion der Argumentation des Schreibers auf den Status quo der militärischen Ausstattung Israels führt daher zu einem die Realität verfälschenden Fehlschluss. Dieser ist jedoch kompatibel mit seinem Israelbild.

(60) „Sie argumentieren, Israel kämpfte um seine 'Existenz'. Zugegeben, das Leben in Israel ist unter diesen Umständen fast unzumutbar. Bedenken Sie aber bitte, Israel hat mehrere tausend Panzer und gepanzerte Fahrzeuge, die zweitstärkste Luftwaffe der Welt, in Extremsituationen zweieinhalb Millionen Soldaten und dazu Atomwaffen, die es -da habe ich keinen Zweifel- auch einsetzen würde. Welches Land könnte einen Krieg gegen Israel riskieren? Herr Präsident, dass Israel um seine Existenz kämpft, ist einfach Unsinn.“ [ZJD_05.04.2002_Stu_001] 
Zum Teil stützen sich die Verfasser bei ihren Beurteilungen auf reine Spekulationen und Glaubenssätze, die sie jedoch als „faktische Argumente“ umdeuten und anführen:

$$
\begin{aligned}
& \text { „Die Erfahrung zeigt doch, daß mit jedem getöteten ‘Terroristen’ zwei } \\
& \text { neue geboren werden!! [...] dann betreiben Sie aber Staatsterrorismus, } \\
& \text { und stellen sich auf die gleiche Stufe wie die von Ihnen bekämpften Ter- } \\
& \text { roristen.“[IBD_23.03.2003_Sch_001] }
\end{aligned}
$$

Mit Rückgriff auf die Topos-Stütze 'Erfahrung' wird die These aufgestellt, jeder getötete Terrorist würde zwei neue hervorbringen. Für diese These gibt es keine empirischen Erfahrungswerte, sie entspringt dem Glaubenssystem des Produzenten aus Hersbruck (und basiert mutmaßlich auf dem Floskelsatz Gewalt produziert nur Gegengewalt). Dem Verfasser dient sie aber maßgeblich als Stütze für seine ablehnende Haltung gegenüber israelischer Militärgewalt (auch im Fall von Gegen- oder Notwehr). Die auf diesem Argument geschlossene Konklusion, Israel betreibe Staatsterrorismus, weil es sich mittels Militäraktionen gegen Terrorismus wehre und damit die Gewalt verdoppele, widerspricht allen Regeln der Vernunft (und des Völkerrechts) und ergibt nur durch das Anlegen eines besonderen (unikalen) Status an Israel ${ }^{21}$ Sinn, klingt aber für den Textproduzenten offensichtlich plausibel und überzeugend. So simulieren die Verfasser gemäß ihrer juden- und israelfeindlichen Konzeptualisierungen Plausibilität und Rationalität, die de facto nicht existieren, bleiben so aber konsistent im mentalen System ihrer Überzeugung.

In Kontrast zur Irrationalität der mentalen Konstrukte steht folglich deren kommunikative Verwendung, d.h. ihre Verbalisierung und textuelle Einbettung. ${ }^{22}$ Akribisch versuchen die Schreiber, ihre Meinung über Juden als empirisch begründet und argumentativ schlüssig hergeleitet darzustellen. So bilden Stereotype und vermeintliche Fakten, Argumente und Belege, die sich gegenseitig bedingen bzw. stützen, ein komplexes argumentatives Netz judenfeindlicher Pseudo-Logik.

Zusammenfassend lässt sich festhalten, dass in den Schreiben (auch von sehr gebildeten Verfassern) aus argumentationstheoretischer Sicht oft die expliziten Stützen oder Schlussregeln für vorgebrachte Argumente fehlen bzw. anfecht-

21 Zur unikalen Bewertung (Doppelstandard) von Israel s. Kap. 7.2.3. Hier ergibt sich diese einmalige Bewertung dadurch, dass Israel, im Gegensatz zu allen anderen Staaten der Erde, das Recht abgesprochen wird, gegen Terrorismus vorzugehen.

22 Hacker (1990: 33) bezeichnet dieses Phänomen treffend als eine „sich rational gebende Verwendung irrationaler Konzepte“. 
bar sind, teils weil die Produzenten rein emotional-irrational und auf der Basis geschlossener Weltbilder argumentieren und teils, weil sie unzulässige Prämissen zur Untermauerung ihrer Argumente heranziehen. Dabei bilden oft anti-jüdische Stereotype die mentale Basis für Prämissen bzw. Stützen. Logische Brüche und Trugschlüsse treten auf, wenn aus solchen Prämissen bestimmte Schlussfolgerungen abgeleitet werden (und zwar nicht aufgrund einer inhärenten Logik, sondern aufgrund von Erwünschtheit der von den Produzenten erzwungenen Konklusionen). Diese ähneln damit den selbsterfüllenden Prophezeiungen, d. h. sie entsprechen exakt den Erwartungen der Sprachproduzenten, weil sie aus deren Sichtweise heraus als plausibel und real konstruiert wurden. So produzieren antisemitische Textproduzenten, der inneren „Logik“ ihres Bewertungssystems folgend, ihre eigenen Bestätigungen für ihr judeophobes Ressentiment.

\subsubsection{Widersprüche und Paradoxien: Aussagen in Kollision}

Neben ungültigen Argumentationsketten und falschen Behauptungen innerhalb der konstruierten Begründungszusammenhänge kommt es in den Zuschriften auch oft zu absurden inhaltlichen oder logischen Widersprüchen. Ein Widerspruch liegt vor, wenn ein Sprachproduzent in seinem Schreiben artikuliert, dass etwas der Fall ist und dass dieses nicht der Fall ist (nach dem Muster ' $P$ ist und $P$ ist nicht'). Hieraus ergeben sich Paradoxien, die den Gesetzen des Verstandes zuwiderlaufen. Unvereinbare Aussagen zeigen sich besonders oft nach Selbstattribuierungen ${ }^{23}$ und -legitimierungen wie „Ich bin ein Humanist“ oder „Ich bin kein Antisemit“, die in eklatantem Kontrast zu den weiteren (antisemitischen) Aussagen der Schreiber stehen. In (62) bescheinigt sich der Verfasser zunächst selbst, kein Antisemit zu sein, um dann in zahlreichen, Juden und Israelis gleichermaßen mittels Dehumanisierungen diskreditierenden Äußerungen diese Aussage selbst ostentativ zu widerlegen:

23 Auch halten es viele Textproduzenten offensichtlich nicht für notwendig, ihre Anschreiben - die sie immerhin an Vertreter offizieller Institutionen wie den Botschafter, den Zentralratsvorsitzenden oder auch (in Kopie) an Regierungsvertreter wie Minister oder die Bundeskanzler(in) richten - vor dem Absenden nochmals zu prüfen, zu korrigieren oder zu revidieren. Auch die Vielzahl an Rechtschreibfehlern und falschen grammatischen Bezügen belegt die mangelnde reflexive Kontrolle, die angesichts der selbstbescheinigten Bildung und Informiertheit oder der signalisierten „aufgezwungenen“ Zurückhaltung zu erwarten wäre. 
„Ich bin deutscher Staatsbürger und kein Antisemit [...] Kreaturen aus Israel, die sich Menschen nennen, schlachten unschuldige Kinder und Frauen ab. [...] Ihr seid einfach nur menschlicher Müll.“ [IBD_20.02.2009_Wei_001]

Diese in den Texten des Korpus vielfach auftretenden Widersprüche gehen mit einer auffälligen Diskrepanz zwischen Selbst- und Fremdwahrnehmung einher und basieren oft auf der Konzeptualisierung, dass Juden nicht mit den gleichen Maßstäben zu beurteilen seien, die für alle andern Menschen gelten.

„dazu gehört auch die tolteranz und der respekt vor fremden ebenso wie das eintreten für frieden und menschenrechte in der welt. [...] das was die menschenfresser in der israelischen regierung [...] anrichten, ist terror gegen die menschlichkeit.“[ZJD_12.04.2002_Wie_001]

Solche semantischen Oppositionen und Inkompatibilitäten ergeben sich auch in Äußerungen wie (64) und (65):

(64) „Ein Menschenfreund der die Juden abgrundtief haßt!“

[ZJD_Gaza2009_792/816_ano_001]

(65) „Ich bin kein Rassist und achte die Menschen. [...] Halten sie sich für was besonderes ausser das sie Massenmörder sind? Ich bete zu gott das in meiner Familie kein JUDE auftaucht.“

[ZJD_Gaza_2009_295/816_Ado_001]

Der Verfasser von (64) widerlegt bereits in der Prädikation desselben Satzes seine Selbstreferenzialisierung, ein Menschenfreund zu sein. Auf die Konzeptualisierung in seinem Kopf zurückgreifend, dass Juden keine Menschen seien, ist für ihn aber kein Widerspruch vorhanden. Die Verfasserin von (65) leugnet zunächst eine rassistische Einstellung und stellt sich selbstlegitimierend als jemand dar, der Respekt vor allen Menschen habe, führt aber dann mit großer emotionaler Emphase genau diese Aussagen ad absurdum, indem sie ihrer rassistischen Kollektiv-Ablehnung gegenüber Juden Ausdruck verleiht.

In (66) erklärt der (akademische) Schreiber zunächst seine Vorurteilsfreiheit und leugnet Ablehnungstendenzen und negative Gefühle gegenüber Juden.

(66) „Ich bin Deutscher und stolz darauf. Mein Geburtsjahr ist 1951 und ich habe mit dem Nationalsozialismus nichts zu tun. Meine Generation hat den Juden niemals etwas getan und wird es auch nicht. Wir haben nichts 
gegen Juden. [...] Ihr Verhalten erzeugt nur weiteren Hass zwischen meiner Generation und den Juden.“ [ZJD_09.11.2008_Det_001]

Diese Aussagen widersprechen jedoch der (stereotypbelasteten) Behauptung, der Zentralrat sei verantwortlich für den (vorher als nicht existent deklarierten) Hass zwischen Juden und Deutschen.

Die Produzentin von (67) verkündet zunächst die Meinung, der Nahostkonflikt gehe keinen Deutschen etwas an, und es sei besser, sich nicht einzumischen. Es folgen dann aber über viele Seiten hinweg Kommentare, utopische Lösungsvorschläge und Belehrungen, die diese zuvor getroffene Feststellung negieren.

„Der Krieg in Israel ist doch nichts was uns Deutsche irgendetwas angeht. Ich bin auch absolut dagegen, dass wir uns da mit einmischen sollen. [...] Meine Kritik an [...] Vielleicht sollten die europäischen Juden doch auf ihr Land Einfluss nehmen um diesen unmöglichen und sinnlosen Krieg zu beenden.“[ZJD_Gaza2009_238/816_Kla_001]

Strukturell ähnlich ist die E-Mail (68):

„Ich schreibe Ihnen obwohl ich nicht viel Ahnung von Juden habe. ...Für mich gibt es nur Menschen. Rassenunterschiede kenn ich nicht. .Mittlerweile verstehe ich die Judenhasser.In meiner Familie gibt es Polen Russen,Inder usw. Ich möchte keinen Juden in der Familie haben. Diese Schande überlebe ich nicht.“ [ZJD_Gaza2009_349/816_Mah_001]

Obwohl die E-Mail-Schreiberin nach eigenen Angaben „nicht viel Ahnung von Juden" hat, kodiert sie in ihren danach folgenden langen Ausführungen ein Stereotyp nach dem anderen (s. Kap. 9.5). Trotz der eingeräumten Wissensdefizite ist sie überzeugt zu „wissen“, dass „Juden verkommen sind“ und in Israel „Massenmord begehen". Zugleich verfängt sie sich in einem weiteren Widerspruch: Sie beschreibt sich einerseits selbst als tolerant und vorurteilsfrei, kodiert andererseits aber eine extreme Judenfeindschaft. Ihre „anti-rassistische Toleranz“ vermag sie bei Juden nicht zu praktizieren und lässt dadurch die tradierte und besonders in der NS-Zeit kommunizierte Konzeptualisierung von Juden als DEN PRINZIPIELL ANDEREN, den außerhalb der Wertegemeinschaft der Menschen stehenden Juden transparent werden.

Widersprüchlichkeit entsteht so als Folge der Negation des zuvor Artikulierten, d. h. wenn eine auf sich selbst anwendbare Aussage negiert wird. In (69) ergibt sich ein ostentativer inhaltlicher Widerspruch dadurch, dass die Verfasserin, eine promovierte Historikerin, genau das tut, was sie zuvor abgelehnt bzw. 
von sich gewiesen hat: nämlich die Rechtfertigung von palästinensischen Terroranschlägen.

„Ich bin weit davon entfernt, die Selbstmordattentate [= der Palästinenser, d. Verf.] zu rechtfertigen [...]. Aber die Selbstmordatentate sind die verzweifelte, teilweise von religiösem Wahn geprägte Reaktion auf eine jahrzehntelange israelische Politik der Vertreibung und permanenten Demütigung der Palästinenser.“ [ZJD_12.04.2002_Hei_001]

Dass verschiedene Aussagen in ein und derselben Zuschrift miteinander unvereinbar sind und dies den Produzenten (offensichtlich) nicht auffällt, deutet auf die konzeptuelle Geschlossenheit der Schreiber hin (bzw. auf ihr Bedürfnis, das positive Selbstbild unter allen Umständen aufrecht zu erhalten).

Die Widersprüche, die in den Briefen an den ZJD und die IBD häufig anzutreffen sind, finden sich auch in zahlreichen Internetkommentaren und judenfeindlichen Publikationen (die mutmaßlich nicht schnell und unreflektiert wie evtl. einige der E-Mails im Korpus verfasst wurden). Eine hierfür exemplarische Textstelle aus einem Pamphlet des rechtsextremistischen Verfassers Horst Mahler macht dies deutlich:

$$
\begin{aligned}
& \text { „Sie [= die Juden, d. Verf.] arbeiten bewußt an der Zersetzung der Volks- } \\
& \text { geister und erstreben die Herrschaft über die Völker. Deshalb sind auch } \\
& \text { die Protokolle der Weisen von Zion - auch wenn es sich dabei um eine } \\
& \text { Fälschung handelt - authentische Zeugnisse des jüdischen Geistes.“ } \\
& \text { (Mahler, zit. n. BfV 2005: 12) }
\end{aligned}
$$

Die Bedeutungen von Fälschung und authentisch stehen in einer semantischen Opposition: Was falsch ist, kann nicht authentisch sein, eine als Fälschung akzeptierte Schrift kann nicht nach logisch-rationalen oder plausiblen Kriterien ein authentisches Zeugnis sein. Diese nach normalen Verstandesprinzipien sofort als Widerspruch erkennbare Textstelle entlarvt den nach irrationalen Kriterien arbeitenden Kopf des Verfassers: Alles wird subsumiert unter das antisemitische Weltbild, um dieses zu bestätigen, auch wenn sich dadurch logische Paradoxien und Kohärenzbrüche ergeben. Die als Fälschung bekannten verschwörungstheoretischen Protokolle der Weisen von Zion, ein prototypischer und viel in rechtsextremen Kreisen zitierter antisemitischer Text, werden als Beleg für die unterstellten Weltherrschaftsbestrebungen von Juden herangezogen, obgleich dem Verfasser selbst bewusst ist, dass diese „Protokolle“ gefälscht sind. 


\subsection{Hass ohne reales Referenzobjekt: Jude als Abstraktum}

Für viele Menschen mit erkennbarer antisemitischer Einstellung ist das Lexem Jude nicht ein Konkretum, ${ }^{24}$ sondern ein Abstraktum: Dadurch ist es möglich, alles Negative auf dieses Konzept zu projizieren, ohne in Konflikt mit der anders gearteten Realität zu kommen. Die Abneigung gilt einem mentalen Konstrukt, keinem realen Objekt: ${ }^{25}$

(71) „ich hab noch nie mit Juden zutun gehabt, aber aus irgendeinem Grund mag ich euch nicht.“[ZJD_08.08.2007_Bus_001]

(72) „Wissen Sie, Herr Botschafter, ich kenne nicht Ihre Geschichte, ich habe Sie nie sprechen hören, ich weiß nicht, wer Sie sind und was Sie denken, doch allein, daß Sie ein Amt ausfüllen, um einer solch verblendeten Politik zu dienen, macht mir eine große Abneigung gegen Sie. Aber nicht, weil Sie ein Jude sind, sondern weil Sie kein Jude sind, der nach Gerechtigkeit strebt, wie es Ihr Name verheißt.“[IBD_13.07.2006_Hel_001]

(73) „Ich kenne persönlich keinen einzigen Menschen aus Israel, oder jüdischen Glaubens.“[ZJD_Gaza2009_66/816_Her_001]

In Äußerungen wie (71), (72) und (73) wird expressis verbis artikuliert, was in zahlreichen Abhandlungen zum Antisemitismus bereits erwähnt und von uns erörtert worden ist (s. Kap. 3.4 und 4): Das antisemitische Ressentiment ist auf kein bestimmtes Referenzobjekt in der realen Welt ausgerichtet, sondern bezieht sich auf das im Kopf gespeicherte Konzept JUDE. „Für den Antisemiten bleibt der Jude ein unbekanntes Objekt, er weiß nicht, wovon er spricht“ (Glucksmann 2005: 98).

Wider alle Vernunft ist das judeophobe Ressentiment „ein ganz allgemeiner Wertnegativismus, eine ganz unbegründet erscheinende und scheinbar regellos hervorbrechende, plötzliche haßerfüllte Ablehnung“ (Scheler ${ }^{4} 1955$ : 36), die sich

24 Konkreta zeichnen sich dadurch aus, dass sie ein Referenzpotenzial haben, das sich auf die erfahrbare, wahrnehmbare Realität erstreckt. Die Mitglieder werden unter eine übergeordnete, konkrete Kategorie subsumiert und können dabei viele individuelle Eigenarten aufweisen. Bei Abstrakta besteht kein direkter empirisch erfahrbarer Bezug zur Realität. Es sind Hypostasierungen des menschlichen Geistes. Instanzen von Abstrakta sind nicht mittels der Sinne erfahrbar (s. Schwarz ${ }^{3} 2008$ : 123 f.). Jude ist als Lexem ein Konkretum, aber es hat bei vielen Antisemiten den Status eines Abstraktums, da diese sich nicht auf reale Juden, sondern auf eine geistige Kategorie in ihrem Kopf beziehen.

25 S. hierzu auch Hacker (1990: 46) und Hegener (2006: 19). 
nur aufgrund der lange tradierten, kulturell, kommunikativ und kollektiv-emotional verankerten Judenfeindschaft erklären lässt.

Vermeintlich selbstkritisch ist der akademische Verfasser von (74), er möchte sich kein Urteil über den israelischen Botschafter anmaßen, weil er diesen nicht persönlich kenne. Dies hindert ihn nicht daran, die Geschehnisse in Israel zu beurteilen, obwohl er diese nur aus den Medien vermittelt kennt. Bei der Beurteilung des adressierten Botschafters ist also für den Schreiber nur eine direkte, primäre Erfahrung legitimierend, bei der (für ihn unumstößlichen, unzweifelhaften) Be- bzw. Entwertung Israels aber reicht ihm ungeprüfte mediale Sekundärerfahrung.

„Sie selbst, sehr geehrter Herr Botschafter und sehr geehrte Damen und Herren, kenne ich nicht gut genug. Ich werde Sie auch nicht kennenlernen und kann mir daher kein Urteil über Sie anmaßen. Jedoch kann ich mir eine Meinung bilden und ein persönliches Urteil fällen über die Dinge, die ich in den Medien lese, höre und sehe und über die ich in Gesprächen mit Freunden und Bekannten erfahre. Und mein Urteil über die Regierung Ihres Landes steht ebenso fest wie mein Urteil über einen Präsidenten, der illegale Angriffskriege führt, das Völkerrecht missachtet und den Behörden, die für die Ahndung zuständig sind, mit Militäreinsatz droht, sofern sie ernsthaft ermitteln.“ [IBD_07.03.2007_Mue_001]

$\mathrm{Zu}$ den Widerspruchsphänomenen gehört auch, dass selbst hochgebildete, in wissenschaftlichem Denken trainierte und normalerweise strikt nach rationalen Prinzipien arbeitende Akademiker diese Prinzipien außer Acht lassen, wenn es um die Vor-Verurteilung und Diffamierung von Juden und/oder Israelis geht: So gibt ein Professor im Laufe einer sich über mehrere Tage hinziehenden E-MailKorrespondenz auf die Frage, ob er das Land, das er so sehr verdamme, denn auch persönlich gut genug kenne, um solche extrem diffamierenden Aussagen treffen zu können, als Antwort:

„Ich war noch nie in Israel/Palästina, aber seit einem Vortrag von [...] 2002 in [...] (wo sie von ihrer Unterstützung für die Israel-Boykott-Bewegung berichtete), und erst recht seit dem Libanon-Krieg habe ich mich intensiv mit der Problematik befasst.“[20.12.2009_Has_003]

Auf die Frage, auf welchen Beobachtungen und Primärquellen seine (durchweg de-realisierenden und stereotypbestimmten) Aussagen denn basierten, gibt er eine Rede von Jimmy Carter an. Dieser Schreiber, der als Wissenschaftler nach den Kriterien der intersubjektiven Überprüfbarkeit etc. forscht, verliert, wenn 
es um Israel geht, die Fähigkeit, rational zu denken. Ungeprüft werden Sekundärinformationen übernommen und entgegen der sonstigen Arbeits- und Denkweise wird allein Glaubensinhalten vertraut. Diese Personen tun etwas, was sie in ihrem Beruf nie tun würden, verlieren also ihre Professionalität und ihre Fähigkeit, logisch und klar zu denken. Diese Schreiber sind immun gegen faktische Argumente und sie lassen keinen Zweifel an sich selbst zu. Das Zufällige wird zum Wesentlichen erhoben, das Gelesene und Gehörte ohne eigene Erfahrungswerte als absolute Wahrheit deklariert. Spekulationen, die in das anti-israelische Weltbild passen, erhalten vor kritischen Denkprozessen den Vorrang. Hier zeigt sich eine jahrhundertelange Konstante von Judenfeindschaft: Die Bereitschaft, alle Informationen ${ }^{26}$ in Bezug auf die Schlechtigkeit von Juden zu glauben, so unwahrscheinlich sie auch sein mögen. Dies belegt einen enormen Einfluss der emotional-irrationalen Dimension auf die kognitive Wahrnehmung.

Die ostentativen Widersprüche zeigen die Wirkungs- und Gestaltungsmacht der judenfeindlichen Konzeptualisierungen: Die Verfasser unterliegen dem Zwang, alle Informationen und Aussagen in ihr mentales Modell zu integrieren. Zweifel werden dabei nicht zugelassen und nicht passende Informationen werden zurechtgebogen, dabei bleiben die Gesetze der Logik, des Verstandes und der Plausibilität außen vor.

\section{Fazit}

Für die Erklärung des Phänomens der Judenfeindschaft spielen emotional geprägte Einstellungen eine herausragende Rolle. (Kollektive) Gefühle haben maßgeblich Einfluss auf die Art und Weise, wie Juden mental konzeptualisiert und verbal bewertet werden. Die Antisemitismusforschung steht somit auch vor neuen Herausforderungen insofern, als sie die ausgeklammerte bzw. zu wenig beachtete emotionale Dimension von Judenfeindschaft stärker in ihre Erklärungsansätze integrieren muss.

Viele antisemitische Äußerungen lassen über ihr hohes textuelles Emotionspotenzial erkennen, dass die Sprachproduzenten einen tiefen Hass und Argwohn

26 Entsprechend werden die von den Massenmedien zum Teil monoperspektivierten antiisraelischen Berichte zum Nahostkonflikt sofort geglaubt und persuasiv als autoritative Quelle genannt (s. Kap. 7.3), obgleich die meisten (akademischen) Verfasser sicherlich den Massenmedien, wenn es um andere Themen geht, mindestens so viel Skepsis entgegenbringen wie Luhmann (1996: 9): „Was wir über unsere Gesellschaft, ja über die Welt, in der wir leben, wissen, wissen wir durch die Massenmedien. [...] Andererseits wissen wir so viel über die Massenmedien, dass wir diesen Quellen nicht trauen können.“ 
gegenüber Juden und Judentum hegen, den sie jedoch mittels Umdeutungen, externalen Attributionen und Argumentationen zu rationalisieren und zu legitimieren versuchen. Die Weltsicht von Antisemiten ist durch eine irrationale Selektivität geprägt, d.h. die Wahrnehmungen und die mentalen Wissens- bzw. Glaubenskomponenten werden stets so ausgewählt und strukturiert, dass sie die eigene judeophobe Konzeptualisierung und emotionale Haltung bestätigen bzw. aufrechterhalten können. Dadurch entstehen hermetisch abgeschlossene Wirklichkeitskonstruktionen, die resistent gegenüber Fakten sind, stark de-realisierend, also realitätsverzerrend wirken und wider die Prinzipien von Verstand und Vernunft Denkstrukturen irrational beeinflussen. In den Texten spiegelt sich diese De-Realisierung in Trugschlüssen, Faktenausblendungen und Widersprüchen wider. 